International Journal of Pure and Applied Mathematics

Volume 85 No. 5 2013, 819-832

ISSN: 1311-8080 (printed version); ISSN: 1314-3395 (on-line version)

url: http://www.ijpam.eu

doi: http://dx.doi.org/10.12732/ijpam.v85i5.1

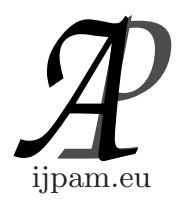

\title{
ON GENERATING FUNCTIONS OF MODIFIED GEGENBAUER POLYNOMIALS
}

\author{
S. Alam ${ }^{1}$, A.K. Chongdar ${ }^{2}$ \\ Department of Mathematics \\ Bengal Engineering and Science University \\ Shibpur, P.O. Botanic Garden, Howrah, 711 103, INDIA
}

\begin{abstract}
In this article, we have obtained some novel theorems on generating functions (both bilateral and mixed trilateral) of modified Gegenbauer polynomials by introducing a partial differential operator obtained by single interpretation to the index of the polynomial under consideration in Weisner's group-theoretic method [2]. Furthermore, we have shown that the results obtained by Das and Chatterjea [1], while investigating some problems on bilateral and mixed trilateral generating functions of Gegenbauer polynomials by using the operator obtained by double interpretations to the index and the parameter simultaneously in Weisner's method, can be easily obtained from our results obtained in this paper. Some applications of our results are also discussed.
\end{abstract}

AMS Subject Classification: $33 \mathrm{C} 47$

Key Words: Gegenbauer polynomials, generating functions, special functions

\section{Introduction}

In the first part of [1], Das and Chatterjea have claimed that the following operator R:

$$
R=\left(x^{2}-1\right) y^{-1} z \frac{\partial}{\partial x}+2 x z \frac{\partial}{\partial y}-x y^{-1} z,
$$

obtained by giving double interpretations to the index (n) and the parameter

Received: November 1, 2008

(C) 2013 Academic Publications, Ltd.

$\S$ Correspondence author url: www.acadpubl.eu 
$(\lambda)$ of the Gegenbauer polynomial in Weisner's group-theoretic method [2], such that

$$
R\left[C_{n}^{\lambda}(x) y^{\lambda} z^{n}\right]=\frac{(n+1)(2 \lambda+n-1)}{2(\lambda-1)} C_{n+1}^{\lambda-1}(x) y^{\lambda-1} z^{n+1}
$$

and

$$
\begin{aligned}
e^{w R} f(x, y, z)= & {\left[1+\frac{2 w x z}{y}+\frac{w^{2} z^{2}\left(x^{2}-1\right)}{y^{2}}\right]^{-\frac{1}{2}} } \\
& f\left(x+\frac{w z\left(x^{2}-1\right)}{y}, y\left\{1+\frac{2 w x z}{y}+\frac{w^{2} z^{2}\left(x^{2}-1\right)}{y^{2}}\right\}, z\right),
\end{aligned}
$$

is original, with the help of which they derived the following generating relation:

$$
\begin{aligned}
{\left[1+4 t x+4 t^{2}\left(x^{2}-1\right)\right]^{\lambda-\frac{1}{2}} } & C_{n}^{\lambda}\left[x+2 t\left(x^{2}-1\right)\right] \\
& =\sum_{m=0}^{\infty}\left(\begin{array}{c}
n+m \\
n
\end{array}\right) \frac{(1-n-2 \lambda)_{m}}{(1-\lambda)_{m}} C_{n+m}^{\lambda-m}(x) t^{m} .
\end{aligned}
$$

Finally, they obtained the following theorems (Theorem 1 - Theorem 3) on generating functions:

Theorem 1. If there exists a generating function of the form

$$
F(x, t)=\sum_{n=0}^{\infty} a_{n} C_{n}^{\lambda-n}(x) t^{n},
$$

then

$$
\begin{aligned}
\sum_{n=0}^{\infty} C_{n}^{\lambda-n}(x) \sigma_{n}(y) t^{n}=\left[1+4 t x+4 t^{2}\left(x^{2}-1\right)\right]^{\lambda-\frac{1}{2}} & \\
& F\left[x+2 t\left(x^{2}-1\right), \frac{y t}{1+4 t x+4 t^{2}\left(x^{2}-1\right)}\right],
\end{aligned}
$$

where

$$
\sigma_{n}(y)=\sum_{k=0}^{n} a_{k}\left(\begin{array}{l}
n \\
k
\end{array}\right) \frac{(1+k-2 \lambda)_{n-k}}{(1+k-\lambda)_{n-k}} y^{k} .
$$

Theorem 2. If there exists a generating function of the form

$$
F(x, y, t)=\sum_{n=0}^{\infty} a_{n} C_{n}^{\lambda-n}(x) g_{n}(y) t^{n}
$$


where $g_{n}(y)$ is an arbitrary polynomial, then

$$
\begin{aligned}
\sum_{n=0}^{\infty} C_{n}^{\lambda-n}(x) \sigma_{n}(y, z) t^{n} & =\left[1+4 t x+4 t^{2}\left(x^{2}-1\right)\right]^{\lambda-\frac{1}{2}} \\
F & {\left[x+2 t\left(x^{2}-1\right), y, \frac{z t}{1+4 t x+4 t^{2}\left(x^{2}-1\right)}\right], }
\end{aligned}
$$

where

$$
\sigma_{n}(y, z)=\sum_{k=0}^{n} a_{k}\left(\begin{array}{l}
n \\
k
\end{array}\right) \frac{(1+k-2 \lambda)_{n-k}}{(1+k-\lambda)_{n-k}} g_{k}(y) z^{k} .
$$

Theorem 3. If there exists a generating function of the form

$$
G(x, t)=\sum_{n=0}^{\infty} a_{n} C_{n}^{\lambda}(x) t^{n}
$$

then

$$
\sum_{n=0}^{\infty} \sigma_{n}(x, t) z^{n}=\left[1+4 t x+4 t^{2}\left(x^{2}-1\right)\right]^{\lambda-\frac{1}{2}} G\left[x+2 t\left(x^{2}-1\right), t z\right],
$$

where

$$
\sigma_{n}(x, t)=\sum_{k=0}^{n} a_{k}\left(\begin{array}{l}
n \\
k
\end{array}\right) \frac{(1-k-2 \lambda)_{n-k}}{(1-\lambda)_{n-k}} C_{n}^{\lambda-(n-k)} t^{k} .
$$

To prove Theorem 1 and Theorem 2, they used the result (1.4) and to obtain Theorem 3 they used the operator directly.

At first, we would like to mention that Das and Chatterjea, while writing the article [1], perhaps fail to notice the work [3]. In fact, the operator R, the extended group generated by R, the generating relation (1.4) and the Theorem 1 obtained by Das and Chatterjea are exactly the same and are found derived earlier in [3].

The aim at presenting this article is to obtain some results (Theorem 4 - Theorem 7) on the bilateral and mixed trilateral generating functions of a modified form of Gegenbauer polynomial by introducing a linear partial differential operator obtained by single interpretation to the index (n) in Weisner's group-theoretic method. Finally, we have shown that the theorems (Theorem 1 - Theorem 3) found derived in [1] can be easily obtained as the particular cases of our results (Theorem 4 - Theorem 6). Here it may be pointed out that the 
operator obtained by single interpretation to the index(n) in the study of modified Gegenbauer polynomials by Weisner's method is very much simple and straightforward even in deriving the nice extensions of the theorems (Theorem 1 - Theorem 3) stated above.

The main results of the present paper are given below:

Theorem 4. If

$$
G(x, t)=\sum_{n=0}^{\infty} a_{n} C_{n+r}^{\lambda-n}(x) t^{n}
$$

then

$$
\begin{aligned}
\sum_{n=0}^{\infty} C_{n+r}^{\lambda-n}(x) f_{n}(v) t^{n}= & {\left[1+4 t x+4 t^{2}\left(x^{2}-1\right)\right]^{\lambda-\frac{1}{2}} } \\
& G\left\{x+2 t\left(x^{2}-1\right), \frac{t v}{1+4 t x+4 t^{2}\left(x^{2}-1\right)}\right\},
\end{aligned}
$$

where

$$
f_{n}(v)=\sum_{m=0}^{n} a_{m}\left(\begin{array}{c}
n+r \\
m+r
\end{array}\right) \frac{(1+m-2 \lambda-r)_{n-m}}{(1+m-\lambda)_{n-m}} v^{m} .
$$

Theorem 5. If

$$
G(x, u, t)=\sum_{n=0}^{\infty} a_{n} C_{n+r}^{\lambda-n}(x) g_{n}(u) t^{n},
$$

then

$$
\begin{aligned}
& \sum_{n=0}^{\infty} C_{n+r}^{\lambda-n}(x) f_{n}(u, v) t^{n}=\left[1+4 t x+4 t^{2}\left(x^{2}-1\right)\right]^{\lambda-\frac{1}{2}} \\
& G\left\{x+2 t\left(x^{2}-1\right), \quad u, \frac{t v}{1+4 t x+4 t^{2}\left(x^{2}-1\right)}\right\},
\end{aligned}
$$

where

$$
f_{n}(u, v)=\sum_{m=0}^{n} a_{m}\left(\begin{array}{c}
n+r \\
m+r
\end{array}\right) \frac{(1+m-2 \lambda-r)_{n-m}}{(1+m-\lambda)_{n-m}} g_{m}(u) v^{m} .
$$

Theorem 6. If

$$
G(x, t)=\sum_{n=0}^{\infty} a_{n} C_{n+r}^{\lambda}(x) t^{n}
$$


then

$$
\sum_{n=0}^{\infty} t^{n} f_{n}(x, z)=\left[1+4 t x+4 t^{2}\left(x^{2}-1\right)\right]^{\lambda-\frac{1}{2}} G\left(x+2 t\left(x^{2}-1\right), z t\right),
$$

where

$$
f_{n}(x, z)=\sum_{k=0}^{n} a_{k}\left(\begin{array}{c}
n+r \\
k+r
\end{array}\right) \frac{(1-k-r-2 \lambda)_{n-k}}{(1-\lambda)_{n-k}} z^{k} C_{n+r}^{\lambda-(n-k)}(x) .
$$

Theorem 7. If

$$
G(x, u, t)=\sum_{n=0}^{\infty} a_{n} C_{n+r}^{\lambda}(x) g_{n}(u) t^{n}
$$

then

$$
\sum_{n=0}^{\infty} \sigma_{n}(x, u, v) t^{n}=\left[1+4 t x+4 t^{2}\left(x^{2}-1\right)\right]^{\lambda-\frac{1}{2}} G\left(x+2 t\left(x^{2}-1\right), u, t v\right),
$$

where

$$
\begin{aligned}
& \sigma_{n}(x, u, v) \\
& \quad=\sum_{k=0}^{n} a_{k}\left(\begin{array}{c}
n+r \\
k+r
\end{array}\right) \frac{(1-k-r-2 \lambda)_{n-k}}{(1-\lambda)_{n-k}} C_{n+r}^{\lambda-n+k}(x) g_{k}(u) v^{k} .
\end{aligned}
$$

In the next section we first proceed to obtain a generating relation by introducing a linear partial differential operator obtained by single interpretation to the index $(\mathrm{n})$ of the polynomial under consideration.

\section{Derivation of a New Generating Function}

At first we consider the following operator $R_{1}$ :

$$
R_{1}=\left(x^{2}-1\right) y \frac{\partial}{\partial x}-2 x y^{2} \frac{\partial}{\partial y}+(2 \lambda-1) x y
$$

such that

$$
R_{1}\left(C_{n+r}^{\lambda-n}(x) y^{n}\right)=\frac{(n+r+1)(1+n-r-2 \lambda)}{2(1+n-\lambda)} c_{n+r+1}^{\lambda-n-1}(x) y^{n+1} .
$$


The extended form of the group generated by $R_{1}$ is given as follows:

$$
\begin{aligned}
e^{w R_{1}} f(x, y)=\left[1+2 w x y+w^{2} y^{2}\left(x^{2}-1\right)\right]^{\lambda-\frac{1}{2}} & \\
& f\left(x+w y\left(x^{2}-1\right), \frac{y}{1+2 w x y+w^{2} y^{2}\left(x^{2}-1\right)}\right) .
\end{aligned}
$$

Now using (2.3), we obtain

$$
\begin{aligned}
& e^{w R_{1}}\left(C_{n+r}^{\lambda-n}(x) y^{n}\right) \\
& \quad=y^{n}\left[1+2 w x y+w^{2} y^{2}\left(x^{2}-1\right)\right]^{\lambda-n-\frac{1}{2}} C_{n+r}^{\lambda-n}\left(x+w y\left(x^{2}-1\right)\right) .
\end{aligned}
$$

But, using (2.2), we obtain

$$
\begin{aligned}
& e^{w R_{1}}\left(C_{n+r}^{\lambda-n}(x) y^{n}\right) \\
& =\sum_{k=0}^{\infty} \frac{w^{k}}{k !} R_{1}^{k}\left(C_{n+r}^{\lambda-n}(x)\right) \\
& =\sum_{k=0}^{\infty} \frac{w^{k}}{k !} \frac{(n+r+1)_{k}(1+n-r-2 \lambda)_{k}}{2^{k}(1+n-\lambda)_{k}} C_{n+r+k}^{\lambda-n-k}(x) y^{k} \\
& =\sum_{k=0}^{\infty}\left(\frac{w y}{2}\right)^{k} \frac{(n+r+1)_{k}(1+n-r-2 \lambda)_{k}}{k !(1+n-\lambda)_{k}} C_{n+r+k}^{\lambda-n-k}(x) .
\end{aligned}
$$

Equating (2.4) and (2.5)and then putting $\frac{w y}{2}=t$, we get

$$
\begin{aligned}
& {\left[1+4 t x+4 t^{2}\left(x^{2}-1\right)\right]^{\lambda-\frac{1}{2}-n} C_{n+r}^{\lambda-n}\left[x+2 t\left(x^{2}-1\right)\right]} \\
& =\sum_{k=0}^{\infty} \frac{(n+r+1)_{k}(1+n-r-2 \lambda)_{k}}{k !(1+n-\lambda)_{k}} C_{n+r+k}^{\lambda-n-k}(x) t^{k} .
\end{aligned}
$$

Now putting $\mathrm{r}=0$ in (2.6), we get

$$
\begin{aligned}
& {\left[1+4 t x+4 t^{2}\left(x^{2}-1\right)\right]^{\lambda-\frac{1}{2}-n} C_{n}^{\lambda-n}\left[x+2 t\left(x^{2}-1\right)\right]} \\
& =\sum_{k=0}^{\infty} \frac{(n+1)_{k}(1+n-2 \lambda)_{k}}{k !(1+n-\lambda)_{k}} C_{n+k}^{\lambda-n-k}(x) t^{k} .
\end{aligned}
$$

which is found derived in [1]. 
Now putting $\mathrm{r}=0$ and replacing $\lambda$ by $\lambda+n$ in (2.6), we get

$$
\begin{aligned}
& {\left[1+4 t x+4 t^{2}\left(x^{2}-1\right)\right]^{\lambda-\frac{1}{2}} C_{n}^{\lambda-n}\left[x+2 t\left(x^{2}-1\right)\right]} \\
& =\sum_{k=0}^{\infty} \frac{(n+1)_{k}(1+n-2 \lambda-2 n)_{k}}{k !(1+n-\lambda-n)_{k}} C_{n+k}^{\lambda-k}(x) t^{k} \\
& =\sum_{k=0}^{\infty}\left(\begin{array}{c}
n+k \\
n
\end{array}\right) \frac{(1-n-2 \lambda)_{k}}{k !(1-\lambda)_{k}} C_{n+k}^{\lambda-k}(x) t^{k}
\end{aligned}
$$

which is (1.4) and found derived in [3].

Now putting $\mathrm{n}=0$ in $(2.8)$, we get

$$
\left[1+4 t x+4 t^{2}\left(x^{2}-1\right)\right]^{\lambda-\frac{1}{2}}=\sum_{k=0}^{\infty} \frac{(1-2 \lambda)_{k}}{k !(1-\lambda)_{k}} C_{k}^{\lambda-k}(x) t^{k}
$$

which is found derived in [3].

Now we proceed to prove the theorems (Theorem 4 - Theorem 7) of this paper.

Proof of Theorem 4. Let us first assume that

$$
G(x, w)=\sum_{n=0}^{\infty} a_{n} C_{n+r}^{\lambda-n}(x) w^{n}
$$

Now replacing $w$ by $w y z$, we have

$$
G(x, w y z)=\sum_{n=0}^{\infty} a_{n}(w z)^{n}\left(C_{n+r}^{\lambda-n}(x) y^{n}\right)
$$

Operating $\exp \left(w R_{1}\right)$ on both sides of (2.11), we get

$$
\begin{aligned}
\left(\exp \left(w R_{1}\right)\right) G(x, & w y z) \\
& =\left(\exp \left(w R_{1}\right)\right)\left\{\sum_{n=0}^{\infty} a_{n}(w z)^{n}\left(C_{n+r}^{\lambda-n}(x) y^{n}\right)\right\} .
\end{aligned}
$$

Now using the results (2.2) and (2.3), we have

$$
\left(\exp \left(w R_{1}\right)\right) G(x, w y z)=\left[1+2 w x y+w^{2} y^{2}\left(x^{2}-1\right)\right]^{\lambda-\frac{1}{2}}
$$




$$
G\left\{x+w y\left(x^{2}-1\right), \frac{w y z}{1+2 w x y+w^{2} y^{2}\left(x^{2}-1\right)}\right\}
$$

and

$$
\begin{aligned}
& \left(\exp \left(w R_{1}\right)\right)\left\{\sum_{n=0}^{\infty} a_{n}(w z)^{n}\left(C_{n+r}^{\lambda-n}(x) y^{n}\right)\right\} \\
= & \sum_{n=0}^{\infty} \sum_{m=0}^{\infty} a_{n}(w z)^{n} \frac{w^{m}}{m !} R_{1}^{m}\left(C_{n+r}^{\lambda-n}(x) y^{n}\right) \\
= & \sum_{n=0}^{\infty} \sum_{m=0}^{\infty} a_{n}(w z)^{n} \frac{w^{m}}{m !} \frac{(n+r+1)_{m}(1+n-r-2 \lambda)_{m}}{2^{m}(1+n-\lambda)_{m}} \\
= & \sum_{n=0}^{\infty} \sum_{m=0}^{\lambda-n-m}(x) y^{n+m} \frac{(y w)^{n+m}}{m !} \frac{(n+r+1)_{m}(1+n-r-2 \lambda)_{m}}{2^{m}(1+n-\lambda)_{m}} C_{n+r+m}^{\lambda-n-m}(x) z^{n} \\
= & \sum_{n=0}^{\infty}\left(\frac{y w}{2}\right)^{n} \sum_{m=0}^{n} a_{n-m} \frac{(n-m+r+1)_{m}(1+n-m-r-2 \lambda)_{m}}{m ! 2^{m}(1+n-m-\lambda)_{m}} \quad(2.14) \\
& C_{n+r}^{\lambda-n}(x)(2 z)^{n} .
\end{aligned}
$$

Now equating (2.13) and (2.14) and then replacing $\frac{w y}{2}$ by $t$ and $2 z$ by $v$, we get

$$
\begin{aligned}
& {\left[1+4 t x+4 t^{2}\left(x^{2}-1\right)\right]^{\lambda-\frac{1}{2}} G\left\{x+2 t\left(x^{2}-1\right), \frac{t v}{1+4 t x+4 t^{2}\left(x^{2}-1\right)}\right\}} \\
& =\sum_{n=0}^{\infty} C_{n+r}^{\lambda-n}(x) f_{n}(v) t^{n}
\end{aligned}
$$

where

$$
f_{n}(v)=\sum_{m=0}^{n} a_{m}\left(\begin{array}{c}
n+r \\
m+r
\end{array}\right) \frac{(1+m-2 \lambda-r)_{n-m}}{(1+m-\lambda)_{n-m}} v^{m} .
$$

This completes the proof of Theorem 4. This theorem was also found derived in [5] by classical method.

Corollary 1. Putting $r=0$ in Theorem 4, we get exactly the Theorem 1 found derived in [1].

Proof of Theorem 5. Let

$$
G(x, u, w)=\sum_{n=0}^{\infty} a_{n} C_{n+r}^{\lambda-n}(x) g_{n}(u) w^{n},
$$


where $g_{n}(u)$ is an arbitrary polynomial of degree $\mathrm{n}$.

Now replacing $w$ by $w y z$, we get

$$
G(x, u, w y z)=\sum_{n=0}^{\infty} a_{n}\left(C_{n+r}^{\lambda-n}(x) y^{n}\right) g_{n}(u)(w z)^{n}
$$

Operating $\exp \left(w R_{1}\right)$ on both sides of (2.16), we get

$$
\begin{aligned}
\left(\exp \left(w R_{1}\right)\right) & G(x, u, w y z) \\
= & \left(\exp \left(w R_{1}\right)\right)\left\{\sum_{n=0}^{\infty} a_{n}\left(C_{n+r}^{\lambda-n}(x) y^{n}\right) g_{n}(u)(w z)^{n}\right\} .
\end{aligned}
$$

The left member of (2.17), with the help of (2.3) becomes

$$
\begin{array}{r}
\left(\exp \left(w R_{1}\right)\right) G(x, u, w y z)=\left[1+2 w x y+w^{2} y^{2}\left(x^{2}-1\right)\right]^{\lambda-\frac{1}{2}} \\
G\left\{x+w y\left(x^{2}-1\right), \quad u, \frac{w y z}{1+2 w x y+w^{2} y^{2}\left(x^{2}-1\right)}\right\} .
\end{array}
$$

Also the right member of (2.17), with the help of (2.2) becomes

$$
\begin{aligned}
& \left(\exp \left(w R_{1}\right)\right)\left\{\sum_{n=0}^{\infty} a_{n}\left(C_{n+r}^{\lambda-n}(x) y^{n}\right) g_{n}(u)(w z)^{n}\right\} \\
= & \sum_{n=0}^{\infty} \sum_{m=0}^{\infty} a_{n}(w z)^{n} \frac{w^{m}}{m !} R_{1}^{m}\left(C_{n+r}^{\lambda-n}(x) y^{n}\right) g_{n}(u) \\
= & \sum_{n=0}^{\infty} \sum_{m=0}^{\infty} a_{n}(w z)^{n} \frac{w^{m}}{m !} R_{1}^{m}\left(C_{n+r}^{\lambda-n}(x) y^{n}\right) g_{n}(u) \\
= & \sum_{n=0}^{\infty} \sum_{m=0}^{\infty} a_{n}(w z)^{n} \frac{w^{m}}{m !} \frac{(n+r+1)_{m}(1+n-r-2 \lambda)_{m}}{2^{m}(1+n-\lambda)_{m}} \\
& C_{n+r+m}^{\lambda-n-m}(x) g_{n}(u) y^{n+m} \\
= & \sum_{n=0}^{\infty} \sum_{m=0}^{\infty} a_{n} \frac{(y w)^{n+m}}{m !} \frac{(n+r+1)_{m}(1+n-r-2 \lambda)_{m}}{2^{m}(1+n-\lambda)_{m}} \\
& C_{n+r+m}^{\lambda-n-m}(x) g_{n}(u) z^{n} \\
= & \sum_{n=0}^{\infty}\left(\frac{y w}{2}\right)^{n} \sum_{m=0}^{n} a_{n-m} \frac{(n-m+r+1)_{m}(1+n-m-r-2 \lambda)_{m}}{m ! 2^{m}(1+n-m-\lambda)_{m}}
\end{aligned}
$$




$$
C_{n+r}^{\lambda-n}(x) g_{n-m}(u)(2 z)^{n} .
$$

Now equating (2.18) and (2.19) and then replacing $\frac{w y}{2}$ by $t$ and $2 z$ by $v$, we get

$$
\begin{aligned}
& {\left[1+4 t x+4 t^{2}\left(x^{2}-1\right)\right]^{\lambda-\frac{1}{2}} G\left\{x+2 t\left(x^{2}-1\right), \quad u, \frac{t v}{1+4 t x+4 t^{2}\left(x^{2}-1\right)}\right\}} \\
& =\sum_{n=0}^{\infty} C_{n+r}^{\lambda-n}(x) f_{n}(u, v) t^{n},
\end{aligned}
$$

where

$$
f_{n}(u, v)=\sum_{m=0}^{n} a_{m}\left(\begin{array}{c}
n+r \\
m+r
\end{array}\right) \frac{(1+m-2 \lambda-r)_{n-m}}{(1+m-\lambda)_{n-m}} g_{m}(u) v^{m} .
$$

This completes the proof of Theorem 5 .

Corollary 2. Putting $r=0$ in Theorem 5, we get the Theorem 2 found derived in [1].

We shall now prove the Theorem 6 and Theorem 7 by using the result (2.8).

Proof of Theorem 6 .

$$
\begin{aligned}
& \sum_{n=0}^{\infty} t^{n} f_{n}(x, z) \\
= & \sum_{n=0}^{\infty} t^{n} \sum_{k=0}^{n} a_{k}\left(\begin{array}{c}
n+r \\
k+r
\end{array}\right) \frac{(1-k-r-2 \lambda)_{n-k}}{(1-\lambda)_{n-k}} z^{k} C_{n+r}^{\lambda-(n-k)}(x) \\
= & \sum_{k=0}^{\infty} a_{k} z^{k} \sum_{n=0}^{\infty}\left(\begin{array}{c}
n+k+r \\
k+r
\end{array}\right) \frac{(1-k-r-2 \lambda)_{n}}{(1-\lambda)_{n}} C_{n+r+k}^{\lambda-k}(x) t^{n+k} \\
= & \sum_{k=0}^{\infty} a_{k}(t z)^{k}\left[1+4 t x+4 t^{2}\left(x^{2}-1\right)\right]^{\lambda-\frac{1}{2}} C_{k+r}^{\lambda}\left(x+2 t\left(x^{2}-1\right)\right) \\
= & {\left[1+4 t x+4 t^{2}\left(x^{2}-1\right)\right]^{\lambda-\frac{1}{2}} \sum_{k=0}^{\infty} a_{k} C_{k+r}^{\lambda}\left(x+2 t\left(x^{2}-1\right)\right)(t z)^{k} } \\
= & {\left[1+4 t x+4 t^{2}\left(x^{2}-1\right)\right]^{\lambda-\frac{1}{2}} G\left(x+2 t\left(x^{2}-1\right), z t\right) }
\end{aligned}
$$

which is Theorem 6 . 
Corollary 3. Putting $r=0$ in Theorem 6, we get the Theorem 3 of Das and Chatterjea [1].

\section{Proof of Theorem 7.}

$$
\begin{aligned}
& \sum_{n=0}^{\infty} \sigma_{n}(x, u, v) t^{n} \\
= & \sum_{n=0}^{\infty} t^{n} \sum_{k=0}^{n} a_{k}\left(\begin{array}{c}
n+r \\
k+r
\end{array}\right) \frac{(1-k-r-2 \lambda)_{n-k}}{(1-\lambda)_{n-k}} C_{n+r}^{\lambda-n+k}(x) g_{k}(u) v^{k} \\
= & \sum_{n=0}^{\infty} \sum_{k=0}^{\infty} a_{k}\left(\begin{array}{c}
n+k+r \\
k+r
\end{array}\right) \frac{(1-k-r-2 \lambda)_{n}}{(1-\lambda)_{n}} C_{n+k+r}^{\lambda-n}(x) g_{k}(u) v^{k} t^{n+k} \\
= & \sum_{k=0}^{\infty} a_{k}(v t)^{k} g_{k}(u) \sum_{n=0}^{\infty}\left(\begin{array}{c}
n+k+r \\
k+r
\end{array}\right) \frac{(1-k-r-2 \lambda)_{n}}{(1-\lambda)_{n}} C_{n+k+r}^{\lambda-n}(x) t^{n} \\
= & \sum_{k=0}^{\infty} a_{k}(v t)^{k} g_{k}(u)\left[1+4 t x+4 t^{2}\left(x^{2}-1\right)\right]^{\lambda-\frac{1}{2}} C_{k+r}^{\lambda}\left(x+2 t\left(x^{2}-1\right)\right) \\
= & {\left[1+4 t x+4 t^{2}\left(x^{2}-1\right)\right]^{\lambda-\frac{1}{2}} \sum_{k=0}^{\infty} a_{k}(v t)^{k} C_{k+r}^{\lambda}\left(x+2 t\left(x^{2}-1\right)\right) g_{k}(u) } \\
= & {\left[1+4 t x+4 t^{2}\left(x^{2}-1\right)\right]^{\lambda-\frac{1}{2}} G\left(x+2 t\left(x^{2}-1\right), u t v\right), }
\end{aligned}
$$

which is Theorem 7 and is also found derived in [6] with some mistakes in the statement by using an operator obtained by double interpretations to the $\operatorname{index}(n)$ and parameter $(\lambda)$ of the Gegenbauer polynomial.

Corollary 4. If we put $r=0$ in Theorem 7 , then we get the following result: If

$$
G(x, u, t)=\sum_{n=0}^{\infty} a_{n} C_{n}^{\lambda}(x) g_{n}(u) t^{n}
$$

then

$$
\sum_{n=0}^{\infty} \sigma_{n}(x, u, v) t^{n}=\left[1+4 t x+4 t^{2}\left(x^{2}-1\right)\right]^{\lambda-\frac{1}{2}} G\left(x+2 t\left(x^{2}-1\right), u, t v\right)
$$

where

$$
\sigma_{n}(x, u, v)=\sum_{k=0}^{n} a_{k}\left(\begin{array}{c}
n \\
k
\end{array}\right) C_{n}^{\lambda-n+k}(x) g_{k}(u) v^{k}
$$

which is also found derived as a corollary to the main theorem obtained by double interpretations to the index and parameter of $C_{n}^{\lambda}(x)$ in [6]. 


\section{Applications}

\subsection{Application of Theorem 4}

As an application of Theorem 4, we consider the following generating relation $[3]:$

$$
\begin{aligned}
& \sum_{n=0}^{\infty} \frac{(r+1)_{n}(1-r-2 \lambda)_{n}}{n !(1-\lambda)_{n}} C_{n+r}^{\lambda-n}(x) t^{n} \\
& =\left\{1+4 x t+4 t^{2}\left(x^{2}-1\right)\right\}^{\lambda-\frac{1}{2}} C_{r}^{\lambda}\left(x+2 t\left(x^{2}-1\right)\right) .
\end{aligned}
$$

If in our theorem, we take

$$
a_{n}=\frac{(r+1)_{n}(1-r-2 \lambda)_{n}}{n !(1-\lambda)_{n}},
$$

then

$$
G(x, t)=\left\{1+4 x t+4 t^{2}\left(x^{2}-1\right)\right\}^{\lambda-\frac{1}{2}} C_{r}^{\lambda}\left(x+2 t\left(x^{2}-1\right)\right) .
$$

Therefore by the application of our Theorem 4 we get the following generalization of the result (3.1):

$$
\begin{aligned}
& \sum_{n=0}^{\infty} C_{n+r}^{\lambda-n}\left\{\sum_{m=0}^{n} \frac{(r+1)_{m}(1-r-2 \lambda)_{m}}{m !(1-\lambda)_{m}}\left(\begin{array}{c}
n+r \\
m+r
\end{array}\right) \frac{(1+m-2 \lambda-r)_{n-m}}{(1+m-\lambda)_{n-m}} v^{m}\right\} t^{n} \\
& =Q^{\lambda-\frac{1}{2}}\left\{1+4 x t+4 t^{2}\left(x^{2}-1\right)\right\}^{\frac{1}{2}-\lambda} \\
& C_{r}^{\lambda}\left[x+2 t\left(x^{2}-1\right)+\frac{2 t v\left\{x+2 t\left(x^{2}-1\right)\right\}^{2}-2 t v}{1+4 x t+4 t^{2}\left(x^{2}-1\right)}\right],
\end{aligned}
$$

where

$$
\begin{array}{r}
Q=\left\{1+4 x t+4 t^{2}\left(x^{2}-1\right)\right\}^{2}+4 t v\left\{x+2 t\left(x^{2}-1\right)\right\}\left\{1+4 x t+4 t^{2}\left(x^{2}-1\right)\right\} \\
+4 t^{2} v^{2}\left[\left\{x+2 t\left(x^{2}-1\right)\right\}^{2}-1\right] .
\end{array}
$$

\subsection{Application of Theorem 7}

As an application of our Theorem 7, we consider the following generating relation [7]:

$$
\sum_{n=0}^{\infty} \frac{(r+n) !}{(2 \alpha)_{n}} C_{n+r}^{(\lambda)}(x) C_{n}^{(\alpha)}(u) t^{n}
$$




$$
\begin{aligned}
=(2 \lambda)_{r}(x-u t)^{-2 \lambda-r} F_{4}\left[\lambda+\frac{r}{2}, \lambda+\frac{r}{2}+\frac{1}{2} ; \alpha+\frac{1}{2}, \lambda+\frac{1}{2} ;\right. \\
\\
\left.\quad \frac{\left(u^{2}-1\right) t^{2}}{(x-u t)^{2}}, \frac{x^{2}-1}{(x-u t)^{2}}\right] .
\end{aligned}
$$

If in our Theorem 7, we take

$$
a_{n}=\frac{(r+n) !}{(2 \alpha)_{n}}, \quad g_{n}(u)=C_{n}^{\alpha}(u),
$$

then

$$
\begin{aligned}
& G(x, u, t)=(2 \lambda)_{r}(x-u t)^{-2 \lambda-r} \\
& F_{4}\left[\lambda+\frac{r}{2}, \lambda+\frac{r}{2}+\frac{1}{2} ; \quad \alpha+\frac{1}{2}, \lambda+\frac{1}{2} ; \frac{\left(u^{2}-1\right) t^{2}}{(x-u t)^{2}}, \frac{x^{2}-1}{(x-u t)^{2}}\right] .
\end{aligned}
$$

Therefore by the application our Theorem 7 , we get the following generalization of $(3.4)$

$$
\begin{aligned}
& \sum_{n=0}^{\infty}\left\{\sum_{k=0}^{n} a_{k}\left(\begin{array}{c}
n+r \\
k+r
\end{array}\right) \frac{(1-k-r-2 \lambda)_{n-k}}{(1-\lambda)_{n-k}} C_{n+r}^{\lambda-n+k}(x) g_{k}(u) v^{k}\right\} t^{n} \\
&=(2 \lambda)_{r}\left\{1+4 x t+4 t^{2}\left(x^{2}-1\right)\right\}^{\lambda-\frac{1}{2}}\left\{x+t\left(2 x^{2}-u v-2\right)\right\}^{-2 \lambda-r} \\
& \times F_{4}\left[\lambda+\frac{r}{2}, \lambda+\frac{r}{2}+\frac{1}{2} ; \alpha+\frac{1}{2}, \lambda+\frac{1}{2} ; \frac{\left(u^{2}-1\right) t^{2} v^{2}}{\left\{x+t\left(2 x^{2}-u v-2\right)\right\}^{2}}\right. \\
&\left.\frac{\left\{x+2 t\left(x^{2}-1\right)\right\}^{2}-1}{\left\{x+t\left(2 x^{2}-u v-2\right)\right\}^{2}}\right]
\end{aligned}
$$

\section{References}

[1] S. Das, S.K. Chatterjea, On a partial differential operator for Gegenbauer polynomials, Bull. Cal. Math. Soc., 76 (1984), 351-361.

[2] L. Weisner, Group-theoretic origin of certain generating functions, Pacific Jour. Math., 5 (1955), 1033-1039.

[3] A.K. Chongdar, Group-theoretic study of certain generating functions of Gegenbauer polynomials, Jour. Orissa Math. Soc., 2, No. 2 (1983), 71-85. 
[4] T.I. Sultan, Weisner's methodic survey for Gegenbauer polynomials, Jour. Pure Math., 4 (1984), 79-101.

[5] A.K. Chongdar, M.C. Mukherjee, On a class of bilateral generating relations of certain generating functions, Bull. Inst. Math. Acad. Sinica., 17, No. 1 (1989), 67-73.

[6] A.B. Majumder, On trilateral generating functions of Gegenbauer polynomials, Bull. Cal. Math. Soc., 87 (1995), 449-452.

[7] H.M. Srivastava, J.L. Lavoie, A certain method of obtaining bilateral generating functions, Nederl. Akad. Wetensch. Indag. Math., 37 (1975), 304320 . 